\title{
Correction to: Quantitative Measurement of Cell-Nanoparticle Interactions Using Mass Cytometry
}

\author{
Andrew J. Mitchell, Angela Ivask, and Yi Ju
}

\section{Correction to:}

Chapter 15 in: Helen M. McGuire and Thomas M. Ashhurst (eds.), Mass Cytometry: Methods and Protocols, Methods in Molecular Biology, vol. 1989, https://doi.org/10.1007/978-1-4939-9454-0_15

This chapter was inadvertently published with the acknowledgement section leaving out the following sentence: "This work received funding from South Australian Government PRIF program Project "International Cluster on Nanosafety" of Nicolas H. Voelcker and Enzo Lombi." This correction has been updated in the chapter. 